J. Nonlinear Var. Anal. 3 (2019), No. 2, pp. 225-233

Available online at http://jnva.biemdas.com

https://doi.org/10.23952/jnva.3.2019.2.10

\title{
ON A NEW ALGORITHM FOR SOLVING VARIATIONAL INEQUALITY AND FIXED POINT PROBLEMS
}

\author{
FENGHUI WANG ${ }^{1, *}$, HONGTRUONG PHAM $^{2}$ \\ ${ }^{1}$ Department of Mathematics, Luoyang Normal University, Luoyang 471022, China \\ ${ }^{2}$ Department of Mathematics, Thainguyen University of Economics and Business Administration, \\ Thainguyen 250000, Vietnam
}

\begin{abstract}
The paper is concerned with the problem of finding a common solution of a variational inequality problem governed by Lipschitz continuous monotone mappings and a fixed point problem of nonexpansive mappings. To solve this problem, we introduce a new iterative algorithm which is based on Tseng's extragradient method. Moreover we prove the strong convergence of the algorithm to a solution of the above-stated problem without the hypothesis of the asymptotical regularization.
\end{abstract}

Keywords. Extragradient method; Fixed point; Iterative algorithms; Nonexpansive mapping; Variational inequality.

2010 Mathematics Subject Classification. 47H05, 47H09, 47J20, 49J40.

\section{INTRODUCTION}

Throughout the paper, $\mathscr{H}$ is a real Hilbert space and $C$ is a nonempty closed convex subset of $\mathscr{H}$. A variational inequality problem (VIP) is formulated as a problem of finding a point $x^{*} \in C$ with the property:

$$
\left\langle A x^{*}, z-x^{*}\right\rangle \geq 0, \quad \forall z \in C,
$$

where $A: C \rightarrow \mathscr{H}$ is a single-valued mapping. We denote the solution set of VIP (1.1) by $\operatorname{VI}(C, A)$. A fixed point problem (FPP) is to find a point $\hat{x}$ with the property:

$$
\hat{x} \in C, \quad S \hat{x}=\hat{x},
$$

where $S: C \rightarrow C$ is a nonlinear mapping. The set of fixed points of $S$ is denoted as $\operatorname{Fix}(S)$.

In this paper, we are interested in finding a common solution of VIP (1.1) and of FPP (1.2). Namely, we seek a point $x^{*}$ such that

$$
x^{*} \in \operatorname{Fix}(S) \cap \operatorname{VI}(C, A) .
$$

This problem was first introduced by Takahashi and Toyoda [11]. Since then, many algorithms have been built for approximating a solution of problem (1.3); see, e.g., [3, 4, 8, 9, 13, 14, 16] and the references therein.

${ }^{*}$ Corresponding author.

E-mail address: wfenghui@gmail.com (F. Wang).

Received March 19, 2019; Accepted June 1, 2019.

(C)2019 Journal of Nonlinear and Variational Analysis 
In the case where $A: C \rightarrow \mathscr{H}$ is inverse strongly monotone and $S: C \rightarrow C$ is nonexpansive, Takahashi and Toyoda [11] introduced an algorithm which generates a sequence $\left\{x_{n}\right\}$ by the iterative procedure:

$$
x_{n+1}=\left(1-\alpha_{n}\right) x_{n}+\alpha_{n} S P_{C}\left(x_{n}-\lambda_{n} A x_{n}\right),
$$

where $P_{C}$ is the projection of $C$ onto $\mathscr{H}$. The Halpern-type algorithm:

$$
x_{n+1}=\alpha_{n} u+\left(1-\alpha_{n}\right) S P_{C}\left(x_{n}-\lambda_{n} A x_{n}\right),
$$

where $u \in C$ is fixed, was introduced by Iiduka and Takahashi [3]. In both algorithms (1.4) and (1.5), the sequence $\left\{\alpha_{n}\right\}$ is chosen from the interval $[0,1]$. Under certain assumptions, the sequence $\left\{x_{n}\right\}$ generated by algorithm (1.4) (resp., (1.5)) can be weakly (resp., strongly) convergent to a solution of problem (1.3).

In general, the above algorithm dose not work whenever $A$ is only a $k$-Lipschitz-continuous and monotone mapping. In this situation, the following iterative method:

$$
\left\{\begin{array}{l}
y_{n}=P_{C}\left(x_{n}-\lambda_{n} A x_{n}\right), \\
z_{n}=P_{C}\left(x_{n}-\lambda_{n} A y_{n}\right), \\
x_{n+1}=\left(1-\alpha_{n}\right) x_{n}+\alpha_{n} S z_{n},
\end{array}\right.
$$

where $\lambda_{n} \in(0,1 / k)$ and $\alpha_{n} \in(0,1)$, was proposed by Nadezhkina and Takahashi [7] for solving problem (1.3). It is worth noting that this algorithm is motivated by Korpelevich's extragradient method [5] for solving varational inequalities. For any initial guess $x_{0} \in C$, Korpelevich's extragradient method generates an iterative sequence recursively by

$$
\left\{\begin{array}{l}
y_{n}=P_{C}\left(x_{n}-\lambda A x_{n}\right), \\
x_{n+1}=P_{C}\left(x_{n}-\lambda A y_{n}\right),
\end{array}\right.
$$

where $\lambda \in(0,1 / k)$. Subsequently, Zeng and Yao [16] introduced a Halpern-type algorithm, which generates an iterative sequence recursively by

$$
\left\{\begin{array}{l}
y_{n}=P_{C}\left(x_{n}-\lambda_{n} A x_{n}\right), \\
z_{n}=P_{C}\left(x_{n}-\lambda_{n} A y_{n}\right), \\
x_{n+1}=\alpha_{n} u+\left(1-\alpha_{n}\right) S z_{n},
\end{array}\right.
$$

where $\lambda_{n} \in(0,1 / k)$ and $\alpha_{n} \in(0,1)$. Under some mild assumptions, the sequence $\left(x_{n}\right)$ generated by algorithm (1.6) (resp., (1.7)) can be weakly (resp., strongly) convergent to a solution of problem (1.3).

We note that one sufficient condition for the convergence of algorithm (1.7) is $\left\|x_{n+1}-x_{n}\right\| \rightarrow 0$ (see [16, Theorem 3.1]). However, from a practical point of view, such condition is often difficult to verify. In this paper, we propose a new algorithm for solving problem (1.3) in the case where the governed mapping is only Lipschitz-continuous monotone. The potential advantage of this algorithm is that we can prove its strong convergence without assuming $\left\|x_{n+1}-x_{n}\right\| \rightarrow 0$. Our algorithm is mainly based on Tseng's splitting method [12] for solving variational inequalities. For any initial guess $x_{0} \in C$, Tseng's splitting method generates an iterative sequence recursively by

$$
\left\{\begin{array}{l}
y_{n}=P_{C}\left(x_{n}-\lambda A x_{n}\right) \\
x_{n+1}=P_{C}\left(y_{n}-\lambda\left(A y_{n}-A x_{n}\right)\right)
\end{array}\right.
$$

where $\lambda \in(0,1 / k)$. The paper is organized as follows. In the next section, some useful lemmas are given. In Section 3, we propose our algorithm and prove its strong convergence to a solution of problem (1.3). 


\section{PRELIMINARY AND NOTATION}

We shall use the following notation:

- $x_{n} \rightarrow x$ : strong convergence of $\left(x_{n}\right)$ to $x$;

- $x_{n} \rightarrow x$ : weak convergence of $\left(x_{n}\right)$ to $x$;

- $I$ the identity mapping, and $\omega_{w}\left(x_{n}\right):=\left\{x: \exists x_{n_{j}} \rightarrow x\right\}$.

A mapping $S: C \rightarrow C$ is said to be $k$-Lipschitz continuous if there exists a constant $k>0$ so that

$$
\|S x-S y\| \leq k\|x-y\|, \quad \forall x, y \in C .
$$

In particular, if $k=1$, then we say $S$ is a nonexpansive mapping. A mapping $A: C \rightarrow \mathscr{H}$ is said to be monotone if

$$
\langle A x-A y, x-y\rangle \geq 0, \quad \forall x, y \in C ;
$$

$k$-inverse strongly monotone if there exists a constant $k>0$ so that

$$
\langle A x-A y, x-y\rangle \geq k\|A x-A y\|^{2}, \quad \forall x, y \in C .
$$

We use $P_{C}$ to denote the projection from $\mathscr{H}$ onto $C$, namely, for $x \in \mathscr{H}, P_{C} x$ is the unique point in $C$ with the property:

$$
\left\|x-P_{C} x\right\|=\min _{y \in C}\|x-y\| .
$$

It is well known that $P_{C} x$ is characterized by the inequality:

$$
P_{C} x \in C, \quad\left\langle x-P_{C} x, z-P_{C} x\right\rangle \leq 0, \quad \forall z \in C .
$$

The lemma below is referred to as the demiclosedness principle for nonexpansive mappings (see [2]).

Lemma 2.1 (Demiclosedness principle). Let $T: C \rightarrow \mathscr{H}$ be a nonexpansive mapping with $\operatorname{Fix}(T) \neq \emptyset$. If $\left\{x_{n}\right\}$ is a sequence in $C$ such that $x_{n} \rightarrow x$ and $(I-T) x_{n} \rightarrow y$, then $(I-T) x=y$. In particular, if $y=0$, then $x \in \operatorname{Fix}(T)$.

A multi-valued mapping $T: \mathscr{H} \rightarrow 2^{\mathscr{H}}$ is said to be monotone if $\langle u-v, x-y\rangle \geq 0$, for any $u \in T x, v \in$ $T y$; maximal monotone if its graph

$$
\mathscr{G}(T)=\{(x, y): x \in \mathscr{D}(A), u \in A x\}
$$

is not properly contained in the graph of any other monotone operator. The normal cone to $C$ at $x \in C$ is a multi-valued mapping defined by

$$
N_{C} x=\{w \in \mathscr{H}:\langle x-u, w\rangle \geq 0, u \in C\} .
$$

It is known that $N_{C}$ is maximal monotone, and its resolvent is $P_{C}$, that is, $P_{C}=\left(I+\lambda N_{C}\right)^{-1}$ (see [1, p. 334]). Define a mapping $T: \mathscr{H} \rightarrow 2^{\mathscr{H}}$ by

$$
T x= \begin{cases}A x+N_{C} x, & x \in C, \\ \emptyset, & x \notin C .\end{cases}
$$

Lemma 2.2. Let $T$ be defined as (2.2) and A a single-valued monotone mapping. Then

(i) $\mathscr{G}(T)$ is sequentially weakly-strongly closed, that is, if $u_{n} \in T x_{n}, x_{n} \rightarrow x$, and $u_{n} \rightarrow u$, then $u \in T x$;

(ii) $\operatorname{VI}(C, A)=T^{-1}(0)=\{x \in \mathscr{H}, 0 \in T x\}$; 
(iii) for $\lambda>0, y=P_{C}(x-\lambda A x)$ iff

$$
\frac{x-y}{\lambda}+A y-A x \in T y
$$

in particular, $x=P_{C}(x-\lambda A x)$ iff $x \in \mathrm{VI}(C, A)$.

Proof. (i) It is known (see [1, Pro. 20.33]) that if $T$ is maximal monotone, then its graph $\mathscr{G}(T)$ is sequentially weakly-strongly closed. Since A is single-valued, by [10, Theorem 3], $T$ is maximal monotone and thus the first assertion follows.

(ii) To see this, we note that $0 \in T x=\left(A+N_{C}\right) x$ if and only if $-A x \in N_{C} x$. By the definition of the normal cone, this is equivalent to $\langle A x, x-z\rangle \leq 0, \forall z \in C$.

(iii) Since $P_{C}$ is the resolvent of $N_{C}$, it follows

$$
\begin{aligned}
y=P_{C}(x-\lambda A x) & \Leftrightarrow y=\left(I+\lambda N_{C}\right)^{-1}(x-\lambda A x) \\
& \Leftrightarrow y+\lambda N_{C} y \ni x-\lambda A x \\
& \Leftrightarrow \lambda\left(A+N_{C}\right) y \ni x-y+\lambda(A y-A x) .
\end{aligned}
$$

Particularly, we have $x=P_{C}(x-\lambda A x)$ if and only if $0 \in T x$. By part (ii), we get the desired assertion.

We end this section by some useful lemmas.

Lemma 2.3. [6] Let $\left\{s_{n}\right\}$ be a real sequence that does not decrease at infinity, in the sense that there exists a subsequence $\left\{s_{n_{k}}\right\}$ so that

$$
s_{n_{k}} \leq s_{n_{k}+1} \text { for all } k \geq 0 .
$$

For every $n>n_{0}$ define an integer sequence $\{\tau(n)\}$ as

$$
\tau(n)=\max \left\{n_{0} \leq k \leq n: s_{k}<s_{k+1}\right\}
$$

Then $\tau(n) \rightarrow \infty$ as $n \rightarrow \infty$ and for all $n>n_{0}$

$$
\max \left(s_{\tau(n)}, s_{n}\right) \leq s_{\tau(n)+1} .
$$

Lemma 2.4. [15] Let $\left\{s_{n}\right\}$ be a nonnegative real sequence satisfying

$$
s_{n+1} \leq\left(1-\alpha_{n}\right) s_{n}+\alpha_{n} \varepsilon_{n}
$$

where the sequences $\left\{\alpha_{n}\right\} \subset(0,1)$ and $\left\{\varepsilon_{n}\right\}$ is a real sequence. Then $s_{n} \rightarrow 0$ as $n \rightarrow \infty$ provided that

(i) $\sum \alpha_{n}=\infty, \lim _{n} \alpha_{n}=0$;

(ii) $\varlimsup_{n} \varepsilon_{n} \leq 0$ or $\sum \alpha_{n}\left|\varepsilon_{n}\right|<\infty$.

Lemma 2.5. Let $x, y \in \mathscr{H}$ and let $t, s \geq 0$. Then

(i) $\|x+y\|^{2} \leq\|x\|^{2}+2\langle y, x+y\rangle$;

(ii) $\|t x+s y\|^{2}=t(t+s)\|x\|^{2}+s(t+s)\|y\|^{2}-s t\|x-y\|^{2}$. 


\section{A STRONG CONVERGENCE THEOREM}

We now introduce our iterative algorithm. Take an initial guess $x_{0} \in C$; choose $\left\{\alpha_{n}\right\} \subseteq(0,1),\left\{\beta_{n}\right\} \subseteq$ $(0,1),\left\{\gamma_{n}\right\} \subseteq(0,1)$ and $\left\{\lambda_{n}\right\} \subseteq(0,1 / k)$; and define a sequence $\left\{x_{n}\right\}$ by the iterative procedure:

$$
\left\{\begin{array}{l}
y_{n}=P_{C}\left(x_{n}-\lambda_{n} A x_{n}\right), \\
z_{n}=P_{C}\left(y_{n}-\lambda_{n}\left(A y_{n}-A x_{n}\right)\right), \\
x_{n+1}=\alpha_{n} u+\beta_{n} x_{n}+\gamma_{n} S z_{n},
\end{array}\right.
$$

where $u \in C$ is fixed, $\left\{\alpha_{n}\right\},\left\{\beta_{n}\right\}$ and $\left\{\gamma_{n}\right\}$ are positive real sequence such that $\alpha_{n}+\beta_{n}+\gamma_{n}=1$. To state the convergence of the algorithm, we need the following lemma.

Lemma 3.1. Let $\left\{x_{n}\right\},\left\{y_{n}\right\}$ and $\left\{z_{n}\right\}$ be defined by (3.1). Then, for any solution $z$ to problem (1.3), there holds the inequality:

$$
\left\|z_{n}-z\right\|^{2} \leq\left\|x_{n}-z\right\|^{2}-\left(1-\left(k \lambda_{n}\right)^{2}\right)\left\|y_{n}-x_{n}\right\|^{2}
$$

Proof. Since $P_{C}$ is nonexpansive, we have

$$
\begin{aligned}
\left\|z_{n}-z\right\|^{2}= & \left\|P_{C}\left(y_{n}-\lambda_{n}\left(A y_{n}-A x_{n}\right)\right)-z\right\|^{2} \\
\leq & \left\|y_{n}-z-\lambda_{n}\left(A y_{n}-A x_{n}\right)\right\|^{2} \\
= & \left\|y_{n}-z\right\|^{2}+\lambda_{n}^{2}\left\|A y_{n}-A x_{n}\right\|^{2} \\
& -2 \lambda_{n}\left\langle y_{n}-z, A y_{n}-A x_{n}\right\rangle \\
= & \left\|x_{n}-z\right\|^{2}+\left\|y_{n}-x_{n}\right\|^{2}+\lambda_{n}^{2}\left\|A y_{n}-A x_{n}\right\|^{2} \\
& +2\left\langle x_{n}-z, y_{n}-x_{n}\right\rangle-2 \lambda_{n}\left\langle y_{n}-z, A y_{n}-A x_{n}\right\rangle \\
= & \left\|x_{n}-z\right\|^{2}-\left\|y_{n}-x_{n}\right\|^{2}+\lambda_{n}^{2}\left\|A y_{n}-A x_{n}\right\|^{2} \\
& +2\left\langle y_{n}-z, y_{n}-x_{n}\right\rangle-2 \lambda_{n}\left\langle y_{n}-z, A y_{n}-A x_{n}\right\rangle .
\end{aligned}
$$

Having in mind that $y_{n}=P_{C}\left(x_{n}-\lambda_{n} A x_{n}\right)$, we deduce from (2.1) that

$$
\left\langle y_{n}-z, y_{n}-x_{n}\right\rangle \leq-\lambda_{n}\left\langle y_{n}-z, A x_{n}\right\rangle .
$$

Since $A$ is $k$-Lipschitz continuous, it follows that

$$
\begin{aligned}
\left\|z_{n}-z\right\|^{2} \leq & \left\|x_{n}-z\right\|^{2}-\left\|y_{n}-x_{n}\right\|^{2} \\
& +\lambda_{n}^{2}\left\|A y_{n}-A x_{n}\right\|^{2}-2 \lambda_{n}\left\langle y_{n}-z, A y_{n}\right\rangle \\
\leq & \left\|x_{n}-z\right\|^{2}-\left(1-k^{2} \lambda_{n}^{2}\right)\left\|y_{n}-x_{n}\right\|^{2} \\
& -2 \lambda_{n}\left\langle y_{n}-z, A y_{n}\right\rangle .
\end{aligned}
$$

On the other hand, we observe that

$$
\left\langle y_{n}-z, A y_{n}\right\rangle=\left\langle y_{n}-z, A y_{n}-A z\right\rangle+\left\langle y_{n}-z, A z\right\rangle .
$$

Since $z \in \operatorname{VI}(C, A)$ and $A$ is monotone, this implies $\left\langle y_{n}-z, A y_{n}\right\rangle \geq 0$. Hence the desired inequality (3.2) at once follows.

Below is the convergence of algorithm (3.1). 
Theorem 3.1. Let $A: C \rightarrow \mathscr{H}$ be a $k(>0)$-Lipschitz continuous monotone mapping and $S: C \rightarrow C$ a nonexpansive mapping. Suppose that

(i) $0<\underline{\lim }_{n} \beta_{n} \leq \varlimsup_{n} \beta_{n}<1$;

(ii) $\lim _{n} \alpha_{n}=0, \sum_{n} \alpha_{n}=\infty$;

(iii) $0<\underline{\lim }_{n} \lambda_{n} \leq \varlimsup_{n} \lambda_{n}<1 / k$.

If the solution set of problem (1.3), denoted by $\Omega$, is nonempty, then the sequence $\left(x_{n}\right)$ generated by (3.1) converges strongly to $P_{\Omega}(u)$.

Proof. Let $z:=P_{\Omega}(u)$. We first show that $\left\{x_{n}\right\},\left\{y_{n}\right\}$ and $\left\{z_{n}\right\}$ are bounded. By Lemma 3.1, one has

$$
\left\|z_{n}-z\right\| \leq\left\|x_{n}-z\right\| \text {. }
$$

Thus

$$
\begin{aligned}
\left\|x_{n+1}-z\right\| & =\left\|\alpha_{n} u+\beta_{n} x_{n}+\gamma_{n} S z_{n}-z\right\| \\
& \leq \alpha_{n}\|u-z\|+\beta_{n}\left\|x_{n}-z\right\|+\gamma_{n}\left\|z_{n}-z\right\| \\
& \leq \alpha_{n}\|u-z\|+\left(1-\alpha_{n}\right)\left\|x_{n}-z\right\|,
\end{aligned}
$$

where the last inequality follows from the fact that $\alpha_{n}+\beta_{n}+\gamma_{n}=1$. By induction, we have

$$
\left\|x_{n}-z\right\| \leq \max \left\{\|u-z\|,\left\|x_{0}-z\right\|\right\} .
$$

Then $\left\{x_{n}\right\}$ is bounded and so is $\left\{z_{n}\right\}$. Since $P_{C}$ is nonexpansive, one has

$$
\begin{aligned}
\left\|y_{n}-z\right\| & =\left\|P_{C}\left(x_{n}-\lambda_{n} A x_{n}\right)-P_{C}\left(z-\lambda_{n} A z\right)\right\| \\
& \leq\left\|x_{n}-z-\lambda_{n}\left(A x_{n}-A z\right)\right\| \\
& \leq\left\|x_{n}-z\right\|+\lambda_{n} k\left\|x_{n}-z\right\| \\
& \leq 2\left\|x_{n}-z\right\| .
\end{aligned}
$$

Therefore $\left\{y_{n}\right\}$ is bounded.

We next show the following inequality:

$$
\begin{aligned}
\left\|x_{n+1}-z\right\|^{2} \leq & \left(1-\alpha_{n}\right)^{2}\left\|x_{n}-z\right\|^{2}+2 \alpha_{n}\left\langle u-z, x_{n+1}-z\right\rangle \\
& -\sigma\left(\left\|S z_{n}-x_{n}\right\|^{2}+\left\|y_{n}-x_{n}\right\|^{2}\right),
\end{aligned}
$$

where $\sigma$ is a positive number. Indeed, it follows from Lemmas 2.5 and 3.1 that

$$
\begin{aligned}
\left\|x_{n+1}-z\right\|^{2}= & \left\|\alpha_{n}(u-z)+\beta_{n}\left(x_{n}-z\right)+\gamma_{n}\left(S z_{n}-z\right)\right\|^{2} \\
\leq & \left\|\beta_{n}\left(x_{n}-z\right)+\gamma_{n}\left(S z_{n}-z\right)\right\|^{2}+2 \alpha_{n}\left\langle u-z, x_{n+1}-z\right\rangle \\
= & \beta_{n}\left(\beta_{n}+\gamma_{n}\right)\left\|x_{n}-z\right\|^{2}+\gamma_{n}\left(\beta_{n}+\gamma_{n}\right)\left\|S z_{n}-z\right\|^{2} \\
& -\gamma_{n} \beta_{n}\left\|S z_{n}-x_{n}\right\|^{2}+2 \alpha_{n}\left\langle u-z, x_{n+1}-z\right\rangle \\
\leq & \beta_{n}\left(\beta_{n}+\gamma_{n}\right)\left\|x_{n}-z\right\|^{2}+\gamma_{n}\left(\beta_{n}+\gamma_{n}\right)\left\|z_{n}-z\right\|^{2} \\
& -\gamma_{n} \beta_{n}\left\|S z_{n}-x_{n}\right\|^{2}+2 \alpha_{n}\left\langle u-z, x_{n+1}-z\right\rangle \\
\leq & \left(1-\alpha_{n}\right)^{2}\left\|x_{n}-z\right\|^{2}-\gamma_{n}\left(1-\alpha_{n}\right)\left(1-\left(k \lambda_{n}\right)^{2}\right)\left\|y_{n}-x_{n}\right\|^{2} \\
& -\gamma_{n} \beta_{n}\left\|S z_{n}-x_{n}\right\|^{2}+2 \alpha_{n}\left\langle u-z, x_{n+1}-z\right\rangle .
\end{aligned}
$$


By our hypothesis on $\left\{\lambda_{n}\right\},\left\{\beta_{n}\right\},\left\{\gamma_{n}\right\}$ and $\left\{\alpha_{n}\right\}$, we may assume without loss of generality that there is $\sigma>0$ so that

$$
\gamma_{n} \min \left(\beta_{n},\left(1-\alpha_{n}\right)\left(1-\left(k \lambda_{n}\right)^{2}\right)\right) \geq \sigma, \quad \forall n .
$$

Hence, inequality (3.3) immediately follows. If we let $s_{n}=\left\|x_{n}-z\right\|^{2}$ and

$$
c_{n}=\sigma\left(\left\|S z_{n}-x_{n}\right\|^{2}+\left\|y_{n}-x_{n}\right\|^{2}\right)
$$

then inequality (3.3) has the form:

$$
s_{n+1} \leq\left(1-\alpha_{n}\right)^{2} s_{n}+2 \alpha_{n}\left\langle u-z, x_{n+1}-z\right\rangle-c_{n} .
$$

Finally, we prove $s_{n} \rightarrow 0$ by considering two possible cases on $\left\{s_{n}\right\}$.

CASE 1 . $\left\{s_{n}\right\}$ is eventually decreasing (i.e., there exists $N \geq 0$ such that $\left\{s_{n}\right\}$ is decreasing for $n \geq N$ ). In this case, $\left\{s_{n}\right\}$ must be convergent. In view of (3.4), one has

$$
0 \leq c_{n} \leq M \alpha_{n}+\left(s_{n}-s_{n+1}\right)
$$

where $M>0$ is a sufficient large number. Letting $n \rightarrow \infty$ in the last inequality yields $c_{n} \rightarrow 0$, this implies that $\left\{\left\|S z_{n}-x_{n}\right\|\right\}$ and $\left\{\left\|y_{n}-x_{n}\right\|\right\}$ both converge to 0 . Hence

$$
\begin{aligned}
\left\|z_{n}-x_{n}\right\| & =\left\|P_{C}\left(y_{n}-\lambda_{n}\left(A y_{n}-A x_{n}\right)\right)-P_{C} x_{n}\right\| \\
& \leq\left\|y_{n}-x_{n}-\lambda_{n}\left(A y_{n}-A x_{n}\right)\right\| \\
& \leq\left(1+k \lambda_{n}\right)\left\|y_{n}-x_{n}\right\| \\
& \leq 2\left\|y_{n}-x_{n}\right\| \rightarrow 0,
\end{aligned}
$$

where we use the fact $x_{n} \in C$, the nonexpansive property of $P_{C}$ and the Lipschitz continuity of $A$. By the nonexpansiveness of $S$, we have

$$
\begin{aligned}
\left\|x_{n}-S x_{n}\right\| & \leq\left\|x_{n}-S z_{n}\right\|+\left\|S z_{n}-S x_{n}\right\| \\
& \leq\left\|x_{n}-S z_{n}\right\|+\left\|z_{n}-x_{n}\right\| \rightarrow 0 .
\end{aligned}
$$

Using the demiclosedness principle yields $\omega_{w}\left(x_{n}\right) \subseteq \operatorname{Fix}(S)$.

We next show $\omega_{w}\left(x_{n}\right) \subseteq \operatorname{VI}(C, A)$. Having in mind that $\left\|y_{n}-x_{n}\right\| \rightarrow 0$, we have $\omega_{w}\left(x_{n}\right)=\omega_{w}\left(y_{n}\right)$. Let

$$
v_{n}=\frac{x_{n}-y_{n}}{\lambda_{n}}-\left(A x_{n}-A y_{n}\right) \text {. }
$$

It then follows from Lemma 2.2 that $v_{n} \in\left(A+N_{C}\right)\left(y_{n}\right)=: T\left(y_{n}\right)$. By the Lipschitz continuity, one has $\left\|A x_{n}-A y_{n}\right\| \rightarrow 0$. Hence $v_{n} \rightarrow 0$. Using Lemma 2.2 yields $\omega_{w}\left(x_{n}\right) \subseteq T^{-1}(0)=\mathrm{VI}(C, A)$. Altogether, we have $\omega_{w}\left(x_{n}\right) \subseteq \Omega$. Consequently,

$$
\varlimsup_{n \rightarrow \infty}\left\langle u-z, x_{n+1}-z\right\rangle=\max _{x^{\prime} \in \omega_{w}\left(x_{n}\right)}\left\langle u-z, x^{\prime}-z\right\rangle \leq 0,
$$

where the inequality follows from (2.1). In view of (3.4), one has

$$
s_{n+1} \leq\left(1-\alpha_{n}\right)^{2} s_{n}+2 \alpha_{n}\left\langle u-z, x_{n+1}-z\right\rangle .
$$

We therefore apply Lemma 2.4 to (3.5) to conclude $s_{n} \rightarrow 0$.

CASE 2. $\left(s_{n}\right)$ is not eventually decreasing. Hence, we can find a subsequence $\left\{s_{n_{k}}\right\}$ so that $s_{n_{k}} \leq s_{n_{k}+1}$ for all $k \geq 0$. In this case, we may define an integer sequence $\{\tau(n)\}$ as in Lemma 2.3. Since $s_{\tau(n)} \leq$ 
$s_{\tau(n)+1}$ for all $n>n_{0}$, it follows again from (3.4) that $c_{\tau(n)} \leq M \alpha_{\tau(n)} \rightarrow 0$, so that $\left\|S_{\tau(n)} z_{\tau(n)}-x_{\tau(n)}\right\| \rightarrow 0$ and $\left\|y_{\tau(n)}-x_{\tau(n)}\right\| \rightarrow 0$ as $n \rightarrow \infty$. In a similar way to Case 1 , we deduce that $\omega_{w}\left(x_{\tau(n)}\right) \subseteq \Omega$. Therefore

$$
\varlimsup_{n \rightarrow \infty}\left\langle u-z, x_{\tau(n)}-z\right\rangle \leq 0 .
$$

On the other hand, we note that

$$
\begin{aligned}
\left\|x_{\tau(n)}-x_{\tau(n)+1}\right\| & =\left\|\alpha_{\tau(n)}\left(u-x_{\tau(n)}\right)+\gamma_{\tau(n)}\left(S_{\tau(n)} z_{\tau(n)}-x_{\tau(n)}\right)\right\| \\
& \leq \alpha_{\tau(n)}\left\|u-x_{\tau(n)}\right\|+\gamma_{\tau(n)}\left\|S_{\tau(n)} z_{\tau(n)}-x_{\tau(n)}\right\| \\
& \leq M\left(\alpha_{\tau(n)}+\left\|S_{\tau(n)} z_{\tau(n)}-x_{\tau(n)}\right\|\right) \rightarrow 0,
\end{aligned}
$$

which together with (3.6) yields

$$
\varlimsup_{n \rightarrow \infty}\left\langle u-z, x_{\tau(n)+1}-z\right\rangle \leq 0 .
$$

Since $s_{\tau(n)} \leq s_{\tau(n)+1}$ (see (2.3)), it follows from (3.4) that

$$
s_{\tau(n)} \leq 2\left\langle u-z, x_{\tau(n)+1}-z\right\rangle
$$

for all $n>n_{0}$. This together with (3.7) yields

$$
\varlimsup_{n \rightarrow \infty} s_{\tau(n)} \leq 0 .
$$

Hence $s_{\tau(n)} \rightarrow 0$. Moreover we have

$$
\begin{aligned}
\sqrt{s_{\tau(n)+1}} & =\left\|\left(x_{\tau(n)}-z\right)-\left(x_{\tau(n)}-x_{\tau(n)+1}\right)\right\| \\
& \leq \sqrt{s_{\tau(n)}}+\left\|x_{\tau(n)}-x_{\tau(n)+1}\right\| \rightarrow 0 .
\end{aligned}
$$

Consequently, $s_{n} \rightarrow 0$ follows from (2.3) immediately.

Remark 3.1. In a similar way to [16], we can apply our algorithms for finding a common fixed point of Lipschitz pseudocontractive and nonexpansive mappings, and for finding a common zero for two monotone mappings.

\section{Acknowledgments}

The authors would like to thank the referee for useful suggestions. This work was sponsored by Program for Science \& Technology Innovation Talents in Universities of Henan Province with Grant no. 15HASTIT013.

\section{REFERENCES}

[1] H.H. Bauschke, P.L. Combettes, Convex analysis and monotone operator theory in Hilbert spaces, Springer-Verlag, 2011.

[2] K. Goebel, W.A. Kirk, Topics on Metric Fixed Point Theory, Cambridge University Press, Cambridge, 1990.

[3] H. Iiduka, W. Takahashi, Strong convergence theorems for nonexpansive mappings and inverse-strongly-monotone mappings, Nonlinear Anal. 61 (2005), 341-350.

[4] H. Iiduka, W. Takahashi, Strong convergence theorem by a hybrid method for nonlinear mappings of nonexpansive and monotone type and applications, Adv. Nonlinear Var. Inequal. 9 (2006), 1-10.

[5] G.M. Korpelevich, An extragradient method for finding saddle points and for other problems, Ekonomika i Matematicheskie Metody, 12 (1976), 747-756.

[6] P.E. Maingé, Strong convergence of projected subgradient methods for nonsmooth and nonstrictly convex minimization, Set-Valued Anal. 16 (2008), 899-912. 
[7] N. Nadezhkina, W. Takahashi, Weak convergence theorem by an extragradient method for nonexpansive mappings and monotone mappings, J. Optim. Theory Appl. 128 (2006), 191-201.

[8] N. Nadezhkina, W. Takahashi, Strong convergence theorem by a hybrid method for nonexpansive mappings and Lipschitzcontinuous monotone mappings, SIAM J. Optim. 16 (2006), 1230-1241.

[9] K. Nakajo, W. Takahashi, Strong convergence theorems for nonexpansive mappings and nonexpansive semigroups, J. Math. Anal. Appl. 279 (2003), 372-379.

[10] R.T. Rockafellar, On the maximality of sums of nonlinear monotone operators, Trans. Amer. Math. Soc. 149 (1970) 75-88.

[11] W. Takahashi, M. Toyoda, Weak convergence theorems for nonexpansive mappings and monotone mappings, J. Optim. Theory Appl. 118 (2003), 417-428.

[12] P. Tseng, A modified forward-backward splitting method for maximal monotone mappings, SIAM J. Control Optim. 38 (1998), 431-446.

[13] F. Wang, H.K. Xu, Strongly convergent iterative algorithms for solving a class of variational inequalities, J. Nonlinear Convex Anal. 11 (2010), 407-421.

[14] F. Wang, H.K. Xu, Weak and strong convergence theorems for variational inequality and fixed point problems with Tseng's extragradient method, Taiwanese J. Math. 16 (2012), 1125-1136.

[15] H.K. Xu, Iterative algorithms for nonlinear operators. J. London Math. Soc. 66 (2002), 240-256.

[16] L.C. Zeng, J.C. Yao, Strong convergence theorem by an extragradient method for fixed point problems and variational inequality problems, Taiwanese J. Math. 10 (2006), 1293-1303. 